\title{
Density functional theory analysis of hexagonal close-packed elemental metal photocathodes
}

\author{
Tuo Li, Benjamin L. Rickman, and W. Andreas Schroeder \\ Department of Physics, University of Illinois at Chicago, \\ 845 West Taylor Street, Chicago, Illinois 60607-7059, USA
}

(Received 30 March 2015; published 8 July 2015)

\begin{abstract}
A density function theory based analysis of photoemission from hexagonal close packed (hcp) metals is presented and the calculated values of the rms transverse momentum $\left(\Delta p_{T}\right)$ are in good agreement with the available experimental data on Be [Phys. Rev. Lett. 111, 237401 (2013)] and Mg [Proceedings of LINAC 2002, Gyeongju, Korea (2002)]. The lattice constants and work functions of the hcp metals are also examined and are consistent with the available experimental values. In addition, emission from (0001)-oriented Be is examined due to the presence of a strong surface state.
\end{abstract}

DOI: 10.1103/PhysRevSTAB.18.073401

\section{INTRODUCTION}

The performance of Ultrafast Electron Microscopy (UEM) [1,2], Dynamic Transmission Electron Microscopy (DTEM) [3-6], Free Electron Lasers (FELs) and X-Ray Free Electron Lasers (XFELs) is fundamentally dependent upon the quality in the transverse direction of the electron pulses produced by their front-end laser-driven electron gun. In the transverse spatial dimension, a high quality (high brightness) electron pulse requires a low normalized spatial transverse rms emittance; commonly defined as $\varepsilon_{T}=\Delta x \Delta p_{T} / m_{0} c$, where $\Delta x$ is the rms transverse pulse size, $\Delta p_{T}$ is the rms transverse momentum of the electrons in the pulse, $m_{0}$ is the free electron mass, and the $c$ is the speed of light in vacuum. In propagation through perfect (i.e., nonaberrating) electron optics, the transverse emittance of the electron pulse is conserved [7], which generally implies that its initial value at the photocathode must be minimized to obtain a high brightness electron source. The initial electron beam size is limited by either (i) laser beam focusing constraints (i.e., physical optics), (ii) laserinduced optical damage of the photocathode surface, or (iii) screening of the photo-gun's accelerating field near the photocathode surface [8-11] that causes temporal [12] and spatial [13] electron pulse distortion. It is therefore likely that any major improvements in the performance of research instruments employing pulsed laser-driven electron sources will require a significant reduction in the rms transverse momentum $\Delta p_{T}$ of the front-end electron source.

Prior theoretical analyses [14,15] have provided an expression for the rms transverse momentum for planar

\section{tli27@uic.edu}

Published by the American Physical Society under the terms of the Creative Commons Attribution 3.0 License. Further distribution of this work must maintain attribution to the author $(s)$ and the published article's title, journal citation, and DOI.
PACS numbers: 81.05.Bx, 71.15.Mb, 73.20.At, 73.30.+y

metal photocathodes; $\Delta p_{T 0}=\sqrt{m_{0}(\hbar \omega-\phi) / 3}$, where $\phi$ is the effective work function (including the Schottky effect [16]) and $\hbar \omega$ is the incident photon energy. However, these analyses have assumed a perfect canonical metal—one with isotropic parabolic bands (i.e., a single spherical Fermi surface) and positive dispersions associated with the free electron mass $m_{0}$. Such an approximation for the electronic band structure of metal photocathodes is unrealistic for all but a few elemental metals, perhaps the body-centered cubic Group Ia alkali metals and the face-centered cubic Group Ib noble metals. Most elemental metals are known to have anisotropic Fermi surfaces due to either the energetic position of the Fermi level in the electronic band structure or the intrinsic anisotropy of the metal's crystalline structure. The latter is the subject of this paper; specifically, the hexagonal close-packed (hcp) structure [17-29] which is the most prevalent crystal space group amongst the elemental metals. Further complicating a theoretical evaluation of the rms transverse momentum is the known strong work function anisotropy in hep metals [30-32]. As a result, both the excess photoemission energy and the electronic band structure, which together determine the states that may contribute to emission, are dependent on the $(i j k l)$ emission direction, and hence, so will $\Delta p_{T}$. In fact, one should also expect the transverse momentum distribution of the emitted electrons to be spatially anisotropic for certain orientations of the uniaxial hep crystals.

In this work, we describe a density functional theory (DFT) approach to determining the rms transverse momentum $\Delta p_{T}$ of electrons emitted from hcp elemental metals. Section II outlines the first principles methods, compares the obtained lattice constant ratios with known experimental values, and presents the results of a thin-slab method [33] to evaluate the work function $\phi_{(i j k l)}$. In Sec. III, we reduce the 15 possible hcp elemental metal photocathodes to seven, based on a set of reasonable selection criteria. The DFT-based photoemission analysis is then described in 
Sec. IV, using emission from the (0001) face of $\mathrm{Mg}$ as an example. Thereafter, emission from the most prevalent face of hcp metals is simulated for $\mathrm{Be}, \mathrm{Hf}, \mathrm{Mg}, \mathrm{Sc}, \mathrm{Ti}, \mathrm{Y}$, and $\mathrm{Zr}$, and the theoretical values of $\Delta p_{T}$ are compared to available experimental data for polycrystalline $\mathrm{Be}[34,35]$ and $\mathrm{Mg}$ [36] photocathodes. Finally, in Sec. VI, emission from the (0001) face of these seven hcp metals is discussed, and a more detailed analysis of $\mathrm{Be}(0001)$ photocathodes is presented where a robust surface state [37-41] can contribute to photoemission.

\section{FIRST-PRINCIPLES METHODS: BULK PROPERTIES, BAND STRUCTURE, AND WORK FUNCTION}

Any rigorous theoretical analysis of photoemission from a crystalline material requires a knowledge of the band structure near the Fermi level; in particular, the precise energy-momentum relationships, $E(\vec{k})$, of the occupied states and their associated local density of states, $g(E, \vec{k})$. In addition, knowledge of the photoelectric work function, $\phi_{(i j k l)}$, in the $(i j k l)$ crystalline direction of photoemission from hexagonal close-packed (hcp) crystals is required in order to determine which occupied states may contribute to the emission. In Secs. IV and V below, we present the results of such a photoemission analysis, directed to the evaluation of $\Delta p_{T}$, for a selected set of hcp metals (Sec. III). In this section, we describe the first-principles methods employed to determine the electronic band structure of hcp metals and their orientation dependent work function, both of which are dependent upon accurate values of the $a$ and $c$ hcp lattice constants. The results are compared, therefore, to measured lattice constants and, for the latter, to both experimental and theoretical literature values of $\phi_{(i j k l)}$.

The first step in a first-principles photoemission analysis of hep metals is an evaluation of their electronic band structure. These calculations within DFT employ the PWscf code of the Quantum-ESPRESSO suite [42]. Ultrasoft pseudopotentials within the local density approximation [43] are used for all 15 hcp metals; the results of our analysis are not changed if the generalized gradient approximation (GGA) [44] is used instead. A sampling of $10 \times 10 \times 6$ Monkhorst-Pack [45] set of special $k$-points and Marzari-Vanderbilt smearing [46] with a broadening of 0.02 Ryd is employed and, for the $4 d, 5 d$ and $6 p$ metals, relativistic approximations are included $[47,48]$. In addition, ferromagnetic collinear local spin density approximation (LSDA) has been performed to find the optimized geometry of hcp Co due to its magnetic property [49]. The DFT-based calculations were all initiated using the experimental lattice parameters for each hcp metal and then tested for total energy convergence to a tolerance of $10^{-4} \mathrm{eV}$. Minimization of the total energy then yields the theoretical optimized lattice constants $a$ and $c$ that are compared in Table I to their experimental values.
TABLE I. Calculated lattice constants of the 15 hcp metals compared with the experimental literature values.

\begin{tabular}{|c|c|c|c|c|}
\hline & Experiment (a.u.) & Experiment $c / a$ & DFT (a.u.) & $\mathrm{DFT} c / a$ \\
\hline \multirow[t]{2}{*}{$\mathrm{Be}$} & $a=4.32$ & $1.567[50]$ & $a=4.20$ & 1.590 \\
\hline & $c=6.77$ & & $c=6.68$ & \\
\hline \multirow[t]{2}{*}{$\mathrm{Cd}$} & $a=5.63$ & $1.886[17]$ & $a=5.48$ & 1.896 \\
\hline & $c=10.62$ & & $c=10.39$ & \\
\hline \multirow[t]{2}{*}{$\mathrm{Co}$} & $a=4.74$ & $1.571[28]$ & $a=4.52$ & 1.617 \\
\hline & $c=7.45$ & & $c=7.31$ & \\
\hline \multirow[t]{2}{*}{ Hf } & $a=6.04$ & $1.579[47]$ & $a=5.88$ & 1.594 \\
\hline & $c=9.54$ & & $c=9.37$ & \\
\hline \multirow[t]{2}{*}{$\mathrm{Mg}$} & $a=6.06$ & $1.625[51]$ & $a=5.89$ & 1.642 \\
\hline & $c=9.85$ & & $c=9.67$ & \\
\hline \multirow[t]{2}{*}{ Os } & $a=5.17$ & $1.579[47]$ & $a=5.17$ & 1.571 \\
\hline & $c=8.16$ & & $c=8.12$ & \\
\hline \multirow[t]{2}{*}{$\mathrm{Re}$} & $a=5.22$ & $1.609[52]$ & $a=5.04$ & 1.587 \\
\hline & $c=8.40$ & & $c=8.00$ & \\
\hline \multirow[t]{2}{*}{$\mathrm{Ru}$} & $a=5.10$ & $1.582[47]$ & $a=5.16$ & 1.663 \\
\hline & $c=8.07$ & & $c=8.58$ & \\
\hline \multirow[t]{2}{*}{$\mathrm{Sc}$} & $a=6.24$ & $1.591[53]$ & $a=6.03$ & 1.589 \\
\hline & $c=9.93$ & & $c=9.52$ & \\
\hline \multirow[t]{2}{*}{ Tc } & $a=5.18$ & $1.585[30]$ & $a=5.11$ & 1.624 \\
\hline & $c=8.21$ & & $c=8.30$ & \\
\hline \multirow[t]{2}{*}{$\mathrm{Tl}$} & $a=6.53$ & $1.599[29]$ & $a=6.40$ & 1.608 \\
\hline & $c=10.44$ & & $c=10.29$ & \\
\hline \multirow[t]{2}{*}{$\mathrm{Ti}$} & $a=5.58$ & $1.586[53]$ & $a=5.36$ & 1.612 \\
\hline & $c=8.85$ & & $c=8.64$ & \\
\hline \multirow[t]{2}{*}{$\mathrm{Y}$} & $a=6.89$ & $1.572[53]$ & $a=6.61$ & 1.587 \\
\hline & $c=10.83$ & & $c=10.49$ & \\
\hline \multirow[t]{2}{*}{$\mathrm{Zn}$} & $a=5.01$ & 1.804 [17] & $a=4.83$ & 1.888 \\
\hline & $c=9.04$ & & $c=9.12$ & \\
\hline \multirow[t]{2}{*}{$\mathrm{Zr}$} & $a=6.10$ & 1.595 [47] & $a=5.91$ & 1.636 \\
\hline & $c=9.73$ & & $c=9.67$ & \\
\hline
\end{tabular}

The comparison of the DFT-generated lattice constants with the experimental values in Table I not only shows good agreement, but also reveals a wide range in the $c / a$ lattice constant ratio from 1.567 for Be to 1.886 for $\mathrm{Cd}$. This ratio range is related to electronic state mixing and hence anisotropy in the electronic band structure. In the divalent hcp metals $\mathrm{Be}, \mathrm{Mg}, \mathrm{Zn}$, and $\mathrm{Cd}$ the $p$ valence electron levels are closest to the occupied $s$ levels whereas electrons in the $d$ levels are energetically well separated from both in $\mathrm{Zn}$ and $\mathrm{Cd}$. As a consequence, in $\mathrm{Be}$ and $\mathrm{Mg}$, high-symmetry $s-p$ mixing is linked to a preferred formation of the energy band near the Fermi level, and thus, electronic band structures with close to parabolic dispersion below the Fermi level. However, there is no similarity between the alkali metals close to spherical Fermi surfaces and those of $\mathrm{Be}$ and $\mathrm{Mg}$ [54] due to the latter's noncubic crystal symmetry and the fact that two valence electrons ensure that their Fermi surfaces enclose twice the volume in momentum space. Nonetheless, $\mathrm{Mg}$ with a $c / a$ lattice constant ratio of 1.624 is within $1 \%$ of ideal close packing where $c / a=1.633$. In contrast, for $\mathrm{Cd}$ and $\mathrm{Zn}$ there is a significant mixing contribution from the $d$ states 
which leads to large nonideal $c / a$ ratios of 1.886 and 1.896, respectively. For $\mathrm{Tl}$, the only $6 p$ hcp transition metal, $c / a=1.599$; its Fermi surface consists of three nonspherical structures [55] and its Fermi energy lies in an almost pure $6 p$ band above the top of the $s$ band. According to the extensive investigations by F. Batallan et al. [28], R. A. Deegan [56], and D. G. Pettifor [57], the electronic bands of the other ten $d$-block transition metals [ $3 d$ ( $\mathrm{Co}, \mathrm{Sc}$ and $\mathrm{Ti})$, $4 d(\mathrm{Ru}, \mathrm{Tc}, \mathrm{Zr}$ and $\mathrm{Y})$ and $5 d(\mathrm{Hf}, \mathrm{Re}$ and $\mathrm{Os})]$ are primarily $d$-like and not parabolic near Fermi level. They have low lying $d$ levels leading to a $s-p$ and $d$ synthesized band structures with the $c / a$ ratios from 1.540 to 1.609 . The large variation in the $c / a$ lattice constant ratios and associated electronic band structure details for the hcp elemental metals generates anisotropic Fermi surfaces and energy-momentum relationships $E(\vec{k})$, and thus asymmetric local densities of states $g(E, \vec{k})$, in addition to considerable work function anisotropy, $\phi_{(i j k l)}$-all of which are involved in photoemission for any particular crystal direction.

Knowledge of the photoelectric work function and its anisotropy is the second requirement for our DFT-based photoemission analysis. We evaluate $\phi_{(i j k l)}$ using the thinslab method [58] which requires knowledge of the atomic potentials, their lattice positions in the $\mathrm{ABAB} z$-direction hexagonal stacking (Table I), and the metal's Fermi level acquired from the bulk DFT band structure calculations. In our evaluations of $\phi_{(i j k l)}$, unrelaxed slabs generally consisting of 8-13 atomic layers separated by a $15 \AA$ vacuum region are employed as this is sufficient to ensure that both the vacuum and average crystal potential reach equilibrium. The work function may then be determined from the energetic difference between the Fermi and the vacuum levels to a calculation uncertainty of typically $\pm 0.05 \mathrm{eV}$. We note that even though small surface layer relaxations are expected for both (0001) and (1010) faces [59], the surfaces of the hcp metals are also known to be less likely to reconstruct at low temperatures [60]. Moreover, although the local-density approximation (LDA) can be expected to represent the better choice of exchange-correlation functional if a better match to experimental values of $\phi_{(i j k l)}$ is desired, our calculations indicate that even with surface relaxations, neither the LDA nor the GGA exchange-correlation functional appear to represent a more accurate choice [59]. The results obtained from the unrelaxed thin-slab simulations for work functions of the (0001) and (1010) crystal faces for all $15 \mathrm{hcp}$ elemental metals are shown in Table II, together with available experimental and theoretical values from the literature. The tabulated data clearly shows that the intrinsic anisotropic nature of hcp metals also results in a strong crystal orientation dependence of the work function.

\section{PHOTOCATHODE SELECTION CRITERIA}

Although, in principle, all 15 elemental hcp metals could be employed as photocathodes given the appropriate
TABLE II. Work functions of the 15 hcp metals.

\begin{tabular}{|c|c|c|c|c|}
\hline & Surface & $\phi(\mathrm{eV})$ & Method & $\phi_{\mathrm{DFT}}(\mathrm{eV})$ \\
\hline \multirow[t]{6}{*}{$\mathrm{Be}$} & $(\mathrm{P})^{\mathrm{a}}$ & $3.92[61]$ & Experiment & \\
\hline & (P) & $4.98[62]$ & Experiment & \\
\hline & (0001) & $5.10[27]$ & Experiment & 5.61 \\
\hline & & $5.40[63]$ & Theory & \\
\hline & & $5.62[24]$ & Theory & \\
\hline & $(10 \overline{1} 0)$ & & & 3.88 \\
\hline \multirow[t]{3}{*}{$\mathrm{Cd}$} & (P) & $4.22[62]$ & Experiment & \\
\hline & (0001) & $4.00[64]$ & Experiment & 4.17 \\
\hline & $(10 \overline{1} 0)$ & & & 4.76 \\
\hline \multirow[t]{2}{*}{$\mathrm{Co}$} & (P) & $5.00[62]$ & Experiment & \\
\hline & (0001) & $5.55[65]$ & Experiment & 5.62 \\
\hline \multirow[t]{3}{*}{ Hf } & (P) & $3.90[62]$ & Experiment & \\
\hline & $(0001)$ & & & 4.51 \\
\hline & $(10 \overline{1} 0)$ & & & 3.63 \\
\hline \multirow[t]{4}{*}{$\mathrm{Mg}$} & (P) & $3.66[62]$ & Experiment & \\
\hline & (0001) & $4.05[21]$ & Theory & 3.87 \\
\hline & & $3.70[63]$ & Experiment & \\
\hline & $(10 \overline{1} 0)$ & & & 3.79 \\
\hline \multirow[t]{3}{*}{ Os } & (P) & $4.83[62]$ & Experiment & \\
\hline & $(0001)$ & & & 5.62 \\
\hline & $(10 \overline{1} 0)$ & & & 4.94 \\
\hline \multirow[t]{3}{*}{$\operatorname{Re}$} & (P) & $4.96[62]$ & Experiment & \\
\hline & $(0001)$ & $5.22-5.77[66]$ & Experiment & 5.35 \\
\hline & $(10 \overline{1} 0)$ & $5.75[62]$ & Experiment & 5.67 \\
\hline \multirow[t]{3}{*}{$\mathrm{Ru}$} & (P) & $4.71[62]$ & Experiment & \\
\hline & $(0001)$ & $5.40[67]$ & Experiment & 5.37 \\
\hline & $(10 \overline{1} 0)$ & & & 4.59 \\
\hline \multirow[t]{3}{*}{$\mathrm{Sc}$} & (P) & $3.50[62]$ & Experiment & \\
\hline & $(0001)$ & & & 3.81 \\
\hline & $(10 \overline{1} 0)$ & & & 3.10 \\
\hline \multirow[t]{3}{*}{$\mathrm{Tc}$} & (P) & $4.88[62]$ & Experiment & \\
\hline & $(0001)$ & & & 5.15 \\
\hline & $(10 \overline{1} 0)$ & & & 4.31 \\
\hline \multirow[t]{3}{*}{$\mathrm{Tl}$} & (P) & $3.84[62]$ & Experiment & \\
\hline & $(0001)$ & & & 3.90 \\
\hline & $(10 \overline{1} 0)$ & & & 3.93 \\
\hline \multirow[t]{3}{*}{$\mathrm{Ti}$} & (P) & $4.33[62]$ & Experiment & \\
\hline & $(0001)$ & $4.60( \pm 0.2)[68]$ & Experiment & 4.72 \\
\hline & $(10 \overline{1} 0)$ & & & 3.63 \\
\hline \multirow[t]{3}{*}{$\mathrm{Y}$} & (P) & $3.10[62]$ & Experiment & \\
\hline & $(0001)$ & & & 3.60 \\
\hline & $(10 \overline{1} 0)$ & & & 3.08 \\
\hline \multirow[t]{3}{*}{$\mathrm{Zn}$} & (P) & $4.33[62]$ & Experiment & \\
\hline & $(0001)$ & $4.15[21]$ & Theory & 4.43 \\
\hline & $(10 \overline{1} 0)$ & & & 5.04 \\
\hline \multirow[t]{3}{*}{$\mathrm{Zr}$} & (P) & $4.05[62]$ & Experiment & \\
\hline & $(0001)$ & $4.26-4.51[69]$ & Theory & 4.42 \\
\hline & $(10 \overline{1} 0)$ & & & 3.59 \\
\hline
\end{tabular}

${ }^{\mathrm{a}}(\mathrm{P})=$ Polycrystalline.

UV laser radiation source (i.e., incident photo energy), differences in their physical and chemical characteristics will dictate their utility. Ideally, a photocathode material should possess a work function that allows photoemission with readily available UV laser sources, be robust and 
chemically stable, have a sufficiently high melting point, and be free of magnetic effects:

(i). Work function. Although modern laser systems are capable of generating radiation throughout most of the infrared, visible, and ultraviolet (UV) spectral regions, the most readily accessible wavelengths are the harmonics of solid-state lasers such as Ti:sapphire (emitting at ca. $800 \mathrm{~nm}), \mathrm{Nd}$ :YAG (1064 nm), and Yb:fiber based systems $(\sim 1040 \mathrm{~nm})$. As the efficient generation of harmonics shorter than $\sim 250 \mathrm{~nm}$ is complicated by the availability of suitable UV transparent nonlinear crystals, desired photocathodes have work functions less than $\hbar \omega \approx$ $4.7 \mathrm{eV}$ (i.e., the third harmonic of Ti:sapphire or the fourth harmonic of Nd:YAG). From Table II, one can see that this tends to rule out $\mathrm{Co}, \mathrm{Os}, \mathrm{Re}$, and possibly $\mathrm{Ru}$ and $\mathrm{Tc}$ - elements that also have availability issues.

(ii). Robustness, vapor pressure, and chemical stability. The metal photocathode should also be hard enough to allow for a good surface figure to be attained by polishing (to avoid surface roughness effects [70]), be vacuum compatible, and be sufficiently inert chemically to allow for operation at vacuum pressures greater than $10^{-9}$ Torr (as routinely found in electron microscopes). The hardness criterion rules out thallium, which is an extremely soft metal, while the high vapor pressures of $\mathrm{Cd}$ and $\mathrm{Zn}$ ensure that they are not vacuum compatible if the effects of desorption contamination are to be avoided [71]. Chemical stability is also an important criterion, especially if surface oxidation causes a significant change in the photocathode work function [72]. However, since a $\mathrm{Mg}$ photocathode has been demonstrated [36], the analysis presented here will not enforce restrictions based on chemical reactivity as it assumes pure metal surfaces.

(iii). Melting point and optical damage. Metal photocathodes with melting points above $\sim 1,000 \mathrm{~K}$ are also preferred for short electron pulse generation as laser induced heating $[73,74]$ can be significant. Cadmium and zinc, which have melting points below $600 \mathrm{~K}$, do not meet such a requirement. Of course, this form of optical damage may be preceded by other forms of laser radiation induced damage that are generally dependent upon the optical surface quality of the photocathode.

(iv). Magnetism. Magnetic metals such as $\mathrm{Co}$, and the face-centered cubic metals $\mathrm{Ni}$ and $\mathrm{Fe}$, do not generally perform well as low emittance photocathode materials, primarily because the trajectory of photoemitted electrons is strongly affected by the metals' local surface magnetic field [75].

Based on these selection criteria, we will restrict our analysis of the photoemission characteristics of elemental hcp metal photocathodes to $\mathrm{Be}, \mathrm{Hf}, \mathrm{Mg}, \mathrm{Sc}, \mathrm{Ti}, \mathrm{Y}$, and Zr. The fact that the hcp crystal structure is intrinsically anisotropic, and hence, so is the electronic crystal band structure, immediately suggests that the momentum distribution of electrons photoemitted from the (0001) crystal face should be different to that emitted from the (1010) face. As shown in Secs. IV, V and VI below, this is indeed the case even after accounting for the difference in work function for the two faces (Table II). In general, it is only photoemission from the (0001) face that may generate an electron beam with an isotropic transverse momentum distribution. Consequently, the emission characteristics of $\operatorname{Mg}(0001)$ will first be used to introduce our DFT-based photoemission model.

\section{DFT BASED PHOTOEMISSION MODEL AND $\Delta p_{T}$ ANALYSIS}

From a knowledge of the band structure of the metal photocathode [i.e., the electronic state dispersion $E(\vec{k})$ and local density of states $g(E, \vec{k})]$ and the work function anisotropy $\phi_{(i j k l)}$, it is straightforward to determine the momentum components of the photoemitted electron and the weighted [by $g(E, \vec{k})]$ probability of its emission from a particular crystal face over the work function barrier, $T\left(\vec{p}, \vec{p}_{0}\right)$, where $p_{0}=\hbar k_{0}$ is the momentum of the emitted electron in the vacuum. As the momentum of the incident UV photon is negligible in comparison to the momentum of the electron in the crystal $(\hbar k \gg \hbar \omega / c)$ [34,35], the governing energy-momentum relationship for an instantaneous (one-step) photoemission process involving a virtual optically-excited state is

$$
\hbar \omega+E\left(p_{z}, p_{T}\right)=\phi_{(i j k l)}+\frac{1}{2 m_{0}}\left(p_{z 0}^{2}+p_{T}^{2}\right),
$$

where the Fermi level is defined as zero energy. In Eq. (1), the electron momentum is written in terms of its longitudinal component $\vec{p}_{z}=\hbar \vec{k}_{z}\left(\vec{p}_{z 0}=\hbar \overrightarrow{k_{z 0}}\right)$ perpendicular to the (ijkl) emission surface in the metal (vacuum), and its transverse component $\vec{p}_{T}=\hbar \overrightarrow{k_{T}}$ parallel to the surface which is conserved during the emission process [7]. The flux transmission probability over the work function barrier may be expressed as

$$
T=\frac{m_{z}^{*}}{m_{0}} \frac{4 p_{z} p_{z 0}}{\left(p_{z}+p_{z 0}\right)^{2}} ; \quad \frac{1}{m_{z}^{*}}=\left|\frac{\partial^{2} E}{\partial p_{z}^{2}}\right|,
$$

where the magnitude of the local effective mass in the emission direction, $m_{z}^{*}$, is used to account for both electronlike (positive dispersion) and holelike (negative dispersion) of the emitting band(s). The close relationship between the band dispersion $\mathrm{E}\left(\hbar \vec{k}=\vec{p}_{z}+\vec{p}_{T}\right)$ below the Fermi level in the photocathode material and the photoemitted electron momentum distribution is clearly evident in Eqs. (1) and (2). In particular, if the band dispersion restricts electrons with high values of $p_{T}$ from being emitted (an imaginary $p_{z 0}$ ), then a reduced value of the emitted rms transverse momentum $\Delta p_{T}$ should result.

Figure 1 displays the DFT based photoemission simulation results for emission from the (0001) face of 


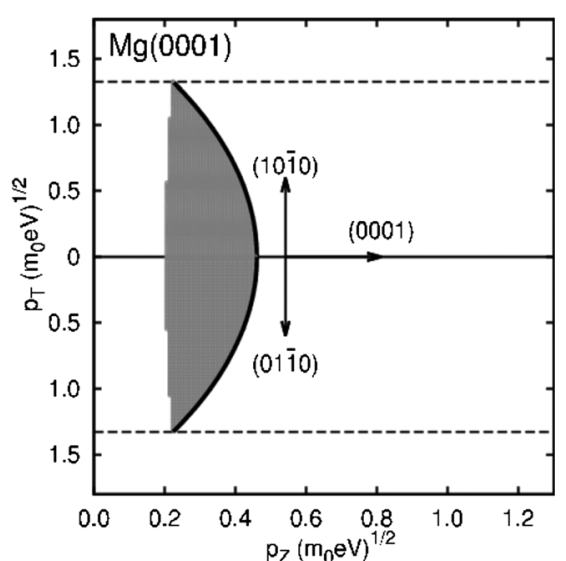

(a)

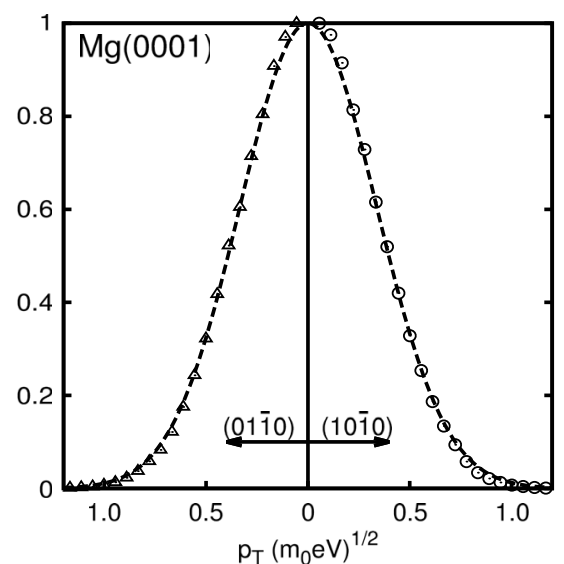

(b)

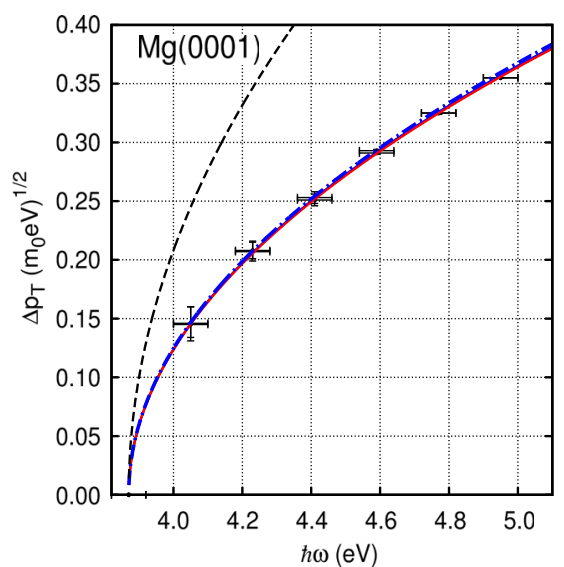

(c)

FIG. 1. $\mathrm{Mg}(0001)$ photoemission analysis for $\hbar \omega=4.75 \mathrm{eV}$. (a) Crystal momentum map of the electronic states (shaded regions) below the Fermi level (solid line) that may photoemit within $p_{T, \max }=\sqrt{2 m_{0} \Delta E}$ (dashed lines) for the transverse (1010) and (0110) crystal directions; (b) Transverse momentum distributions of the photoemitted electrons in the (10) 10$)$ and (01 $\overline{1} 0)$ directions (Gaussian fits are guides to the eye); (c) Incident photon energy dependence of the rms transverse momentum $\Delta p_{T}$ for electron temperatures $T_{e} \rightarrow 0$ (data points with solid red line fit; $\Delta p_{T}=A \sqrt{m_{0} \Delta E}$ ), $T_{e}=300 \mathrm{~K}$ (dot-dashed line), together with the expected form of $\Delta p_{T}(\hbar \omega)$ from Refs. [14] and [15] (dashed line).

magnesium $\left(\phi_{(0001)}=3.87( \pm 0.05) \mathrm{eV}\right)$. Figure 1(a) illustrates the electronic states that contribute to the photoemission (shaded region) below the Fermi level (solid line)

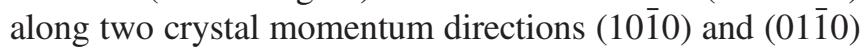
transverse to the (0001) emission direction when the incident photon energy is $4.75 \mathrm{eV}$; that is, for an excess energy $\Delta E=0.88( \pm 0.05) \mathrm{eV}$. As expected from the band structure calculations [51,76], the emission states in the basal plane of this hcp metal are electronlike (positive dispersion) and highly symmetric about the (0001) emission direction for this metal. The dashed line in Fig. 1(a) indicates the maximum possible transverse momentum for the photoemitted electrons, which is simply given by $p_{T, \max }=\left(2 m_{0} \Delta E\right)^{1 / 2} \approx 1.327\left(m_{0} \mathrm{eV}\right)^{1 / 2}$. At this value of the transverse momentum, the transmission efficiency over the photoemission barrier, $T\left(p_{z}, p_{z 0}\right)$, is zero since $p_{z 0}=0$, so that the transverse momentum distributions of the emitted electrons terminate at $p_{T, \max }$. As the transverse momentum $p_{T}$ is reduced, more electron states below the Fermi level can contribute to photoemission with those states nearest the Fermi level having the largest local density of states $g(E, \vec{k})$ and the highest values of $T\left(p_{z}, p_{z 0}\right)$.

The high transverse symmetry of the $\Gamma_{4}^{-}$emission band about the (0001) crystal direction ensures that the resulting weighted transverse momentum distributions of the photoemitted electrons are nearly identical along the (1010) and $(01 \overline{1} 0)$ crystal momentum directions [Fig. 1(b)]. These $p_{T}$ distributions for the emitted electrons in the two crystal directions, which are normalized at $p_{T}=0$, are generated by summing the product $g\left(E, \vec{p}_{z}+\vec{p}_{T}\right) T\left(p_{z}, p_{z 0}\right)$ at discrete values of $p_{T}$ over the electronic states along $p_{z}$ that may contribute to photoemission [i.e., solutions to Eq. (2) with $\left.p_{z 0}>0\right]$. The spatially-averaged value of the rms transverse momentum $\Delta p_{T}$ extracted from these $p_{T}$ distributions for $\operatorname{Mg}(0001)$ emission is $0.322( \pm 0.008)$ $\left(m_{0} \mathrm{eV}\right)^{1 / 2}$ - a value which is only $60 \%$ of that predicted by prior analyses $[14,15] ; \Delta p_{T 0}=0.542( \pm 0.015)$ $\left(m_{0} \mathrm{eV}\right)^{1 / 2}$. The explanation for the lower than expected value of $\Delta p_{T}$ is associated with the energetic position and dispersion of the $\Gamma_{4}^{-}$electron band. Our band structure calculations indicate that the energetic minimum of this band at the $\Gamma$ point is only $1.2 \mathrm{eV}$ below the Fermi level. As a result, for $\Delta E \approx 0.9 \mathrm{eV}$, nearly all the available states along the (0001) crystal direction at $p_{T}=0$ are accessed, generating a gibbous, rather than crescent, shape to the shaded region in Fig. 1(a). Thus, disproportionately more states at lower $p_{T}$ emit than at higher $p_{T}$, which yields a reduced value of $\Delta p_{T}$.

Figure 1(c) displays the dependence of $\Delta p_{T}$ on the incident photon energy $\hbar \omega$ predicted by the DFT-based simulation for photoemission from $\operatorname{Mg}(0001)$. The solid line is a fit to the theoretical data points of the form $\Delta p_{T}=A \sqrt{m_{0}\left(\hbar \omega-\phi_{(0001)}\right)}$, giving a value for $A$ of 0.343 . Comparing this value of $A$ with 0.577 from $\Delta p_{T 0}=$ $\sqrt{m_{0} \Delta E / 3}$ [dashed line in Fig. 1(c)], again indicates that the rms transverse momentum from $\operatorname{Mg}(0001)$ is a factor of $\sim 1.7$ less than expected from prior analyses $[14,15]$. Both of these evaluations of $\Delta p_{T}$ are, of course, determined in the "zero temperature" limit; that is, for an electron temperature $T_{e} \rightarrow 0$, so that no electrons occupy states above the Fermi level. The dot-dashed line (just above the solid line) in Fig. 1(c) shows the predicted form of $\Delta p_{T}(\hbar \omega)$ when $T_{e}=300 \mathrm{~K}$ (data points not shown) which is evaluated by including the Fermi-Dirac function $f(E)=1 /\left(1+\exp \left[-\left(E_{F}-E\right) / k_{B} T_{e}\right]\right)$, where $k_{B}$ 
is Boltzmann's constant, to describe the occupation of the electronic states around the Fermi energy $E_{F}$, albeit for the zero temperature crystal band structure. The increase (or change) in $\Delta p_{T}$ is less than $1 \%$ in this case since $\Delta E$ and the value of $E_{F}$ measured from the bottom of the $\Gamma_{4}^{-}$ band are both much greater than $k_{B} T_{e}$; that is to say that the additional partially populated states above the Fermi level are a very small perturbation in the photoemission simulation. Even if $T_{e}$ is increased to $923 \mathrm{~K}$, the melting point of $\mathrm{Mg}$ [77], the theoretical increase in $\Delta p_{T}$ is insignificant compared to the uncertainties on the DFT-based photoemission simulation. We note that the Boltzmann tail of the Fermi-Dirac distribution will allow photoemission for $\hbar \omega<\phi_{(0001)}$ [73], essentially photoassisted thermionic emission, but this effect is not considered here as the emission efficiency is much reduced.

\section{V. (1010)-FACE EMISSION}

The preferred surface crystal orientation for nearly all hcp polycrystalline metals exposes the (1010) face rather than the (0001) face [78]. Accordingly, in this section, we present the results obtained from the DFT-based simulation for photoemission from the (1010) crystal face of the selected $\mathrm{Be}, \mathrm{Hf}, \mathrm{Mg}, \mathrm{Sc}, \mathrm{Ti}, \mathrm{Y}$, and $\mathrm{Zr}$ elemental metal photocathodes. The thin-slab analysis [33] indicates that $\phi_{(10 \overline{1}))}$ ranges from $3.08 \mathrm{eV}$ for $\mathrm{Y}$ to $3.88 \mathrm{eV}$ for $\mathrm{Be}$ (see Table II). As a result, for incident $4.75 \mathrm{eV}$ (or even $4.67 \mathrm{eV}$ ) UV photons, one might expect efficient $\left(\sim 10^{-4}\right.$ quantum efficiency [36]) isotropic photoemission since the maximum possible transverse momentum $p_{T, \text { max }}=\left(2 m_{0} \Delta E\right)^{1 / 2}$ of around $1.5\left(m_{0} \mathrm{eV}\right)^{1 / 2}$ should allow access to many electronic states in the metal photocathodes. However, as shown below, the highly anisotropic band structure (due to the symmetry of hcp crystals) can play a major role in determining the rms transverse momentum $\Delta p_{T}$ of the photoemitted electrons, even generating beams with a highly elliptical divergence symmetry from the (1010) face.

Figure 2 displays the results obtained from the DFTbased photoemission simulation for the (1010) face of Be, $\mathrm{Mg}$, Y and $\mathrm{Zr}$ when $\hbar \omega=4.75 \mathrm{eV}$. In each case, Fig. 2(a) shows the electronic states (shaded regions) below the Fermi level (solid line) that contribute to photoemission along the (0001) and (0110) crystal momentum directions perpendicular to the (1010) emission direction. An acute asymmetry of the contributing states in transverse momentum $p_{T}$, caused by the energetic position of the Fermi level in the hcp band structure, is immediately evident, with Be being the most asymmetric and $\mathrm{Zr}$ the most symmetric. For $\mathrm{Be}(10 \overline{1} 0)$ emission, the holelike dispersion of the contributing $\Delta_{2}$ band in the (0001) direction is so strong that emission ceases for $p_{T}>0.4\left(m_{0} \mathrm{eV}\right)^{1 / 2}$, even though electron emission up to $p_{T, \max }=1.319\left(m_{0} \mathrm{eV}\right)^{1 / 2}$ (dashed line) from the same band is possible in the $(01 \overline{1} 0)$ momentum direction. The $\Delta_{2}$ band also contributes to the state asymmetry for $\mathrm{Mg}$ emission although to a somewhat lesser extent as some photoemission is now also possible at $p_{T, \max }=1.386\left(m_{0} \mathrm{eV}\right)^{1 / 2}$ (dashed line) in the (0001) transverse momentum direction due to the $T_{2}$ and $T_{4}$ bands [76]. There is also an additional anisotropic contribution to photoemission from $\mathrm{Mg}$ from the electronlike $\Sigma_{1}$ band at lower values of $p_{z}$. For $\mathrm{Y}$, a large number of predominantly holelike electronic states, that are more isotropically distributed about the $(10 \overline{1} 0)$ emission direction than in $\mathrm{Be}$ and $\mathrm{Mg}$ and extend to $p_{T, \text { max }}=1.828\left(m_{0} \mathrm{eV}\right)^{1 / 2}$ (dashed line) in both the transverse (0001) and (0110) crystal momentum directions, will contribute to the photoemission. For the (1010) face of $\mathrm{Zr}$, photoemission with $\hbar \omega=4.75 \mathrm{eV}$ is possible up to $p_{T, \text { max }}=1.523\left(m_{0} \mathrm{eV}\right)^{1 / 2}$ (dashed line) in all transverse directions and the contributing states appear to be even more symmetrically distributed about the (1010) emission direction. The photoemitting states for both $\mathrm{Y}$ and $\mathrm{Zr}$ are terminated by the $M K$ edge of Brillouin zone at $p_{z}=2.93\left(m_{0} \mathrm{eV}\right)^{1 / 2}$ and $p_{z}=3.21\left(m_{0} \mathrm{eV}\right)^{1 / 2}$, respectively.

The trend of decreasing anisotropy from $\mathrm{Be}$, through $\mathrm{Mg}$ and $\mathrm{Y}$, to $\mathrm{Zr}$ is reflected in the transverse momentum distributions of the emitted electrons shown in Fig. 2(b). For example, for $\operatorname{Be}(10 \overline{1} 0)$ emission at the considered $4.75 \mathrm{eV}$ photon energy, the calculated $\mathrm{rms}$ transverse momentum for photoemitted electrons parallel to the (0001) crystal direction, $\Delta p_{T,(0001)}=0.147\left(m_{0} \mathrm{eV}\right)^{1 / 2}$, is a factor of more than two lower than that for the $(01 \overline{1} 0)$ crystal direction, $\Delta p_{T,(01 \overline{1} 0)}=0.312\left(m_{0} \mathrm{eV}\right)^{1 / 2}$. For the (1010)-face of $\mathrm{Mg}$, this ratio in the directional rms transverse momenta of the emitted electrons is reduced to $\sim 1.4$, and it is further reduced for $\mathrm{Y}$ and $\mathrm{Zr}$, with emission from the latter being close to isotropic. All the values of $\Delta p_{T}$ extracted from the DFT analysis are also significantly lower than that expected from the isotropic single-band theory of Dowell and Jensen [14,15]; namely, $\Delta p_{T 0}=\sqrt{m_{0} \Delta E / 3}$ evaluated using the listed values of $\phi_{(10 \overline{10})}$ determined from the thin-slab calculation [33] (from Table II). This is primarily due to the contribution of holelike bands to photoemission for this hep crystal orientation; unlike electronlike bands, holelike states below the Fermi level are generally at higher $p_{T}$ which reduces the available excess energy and hence the transmission efficiency over the work function barrier.

Figure 2(c) displays the photon energy dependence of both $\Delta p_{T,(0001)}$ and $\Delta p_{T,(0110)}$ determined by the DFTbased analysis for (1010)-face emission from the $\mathrm{Be}, \mathrm{Mg}$, $\mathrm{Y}$ and $\mathrm{Zr}$ hcp crystalline photocathodes. The theoretical data points, which reflect the $\pm 0.05 \mathrm{eV}$ uncertainty in the work function evaluation, are well fit by $\Delta p_{T}=$ $A \sqrt{\left(m_{0} \Delta E\right)}$ (solid lines) for electrons emitted with $p_{T}$ in both the (0001) and (1010) crystal directions. Also shown by the dashed lines is the trend in $\Delta p_{T 0}(\hbar \omega)$ from the analysis of Dowell and Jensen $(A=0.577)[14,15]$ which, in all four cases, predicts a value of the rms transverse momentum that is on average a factor of 2 

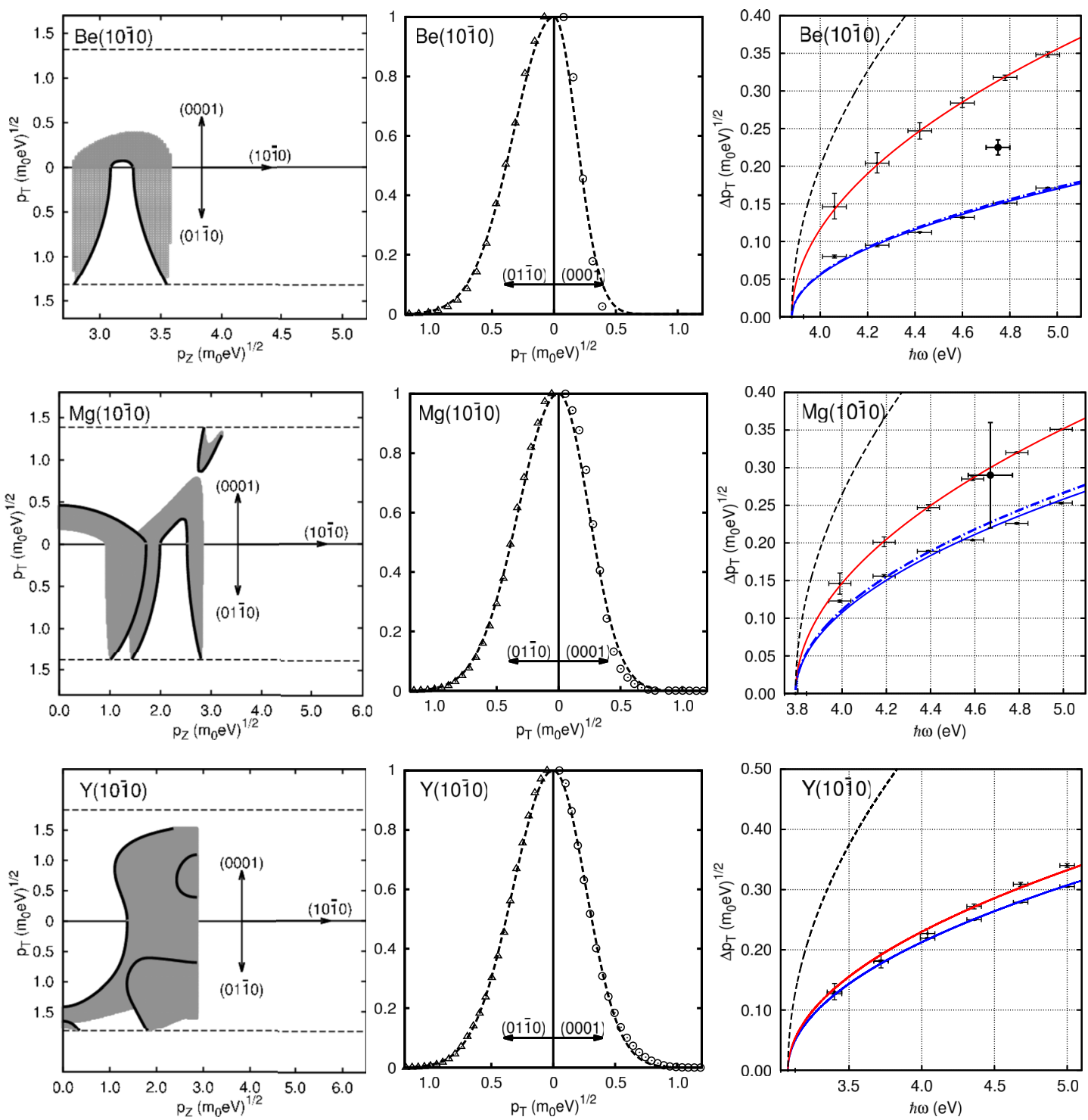

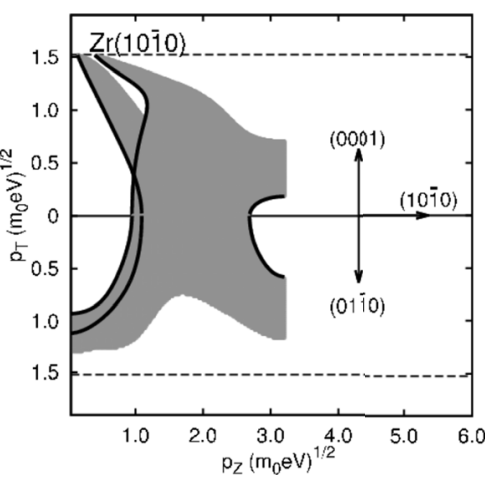

(a)

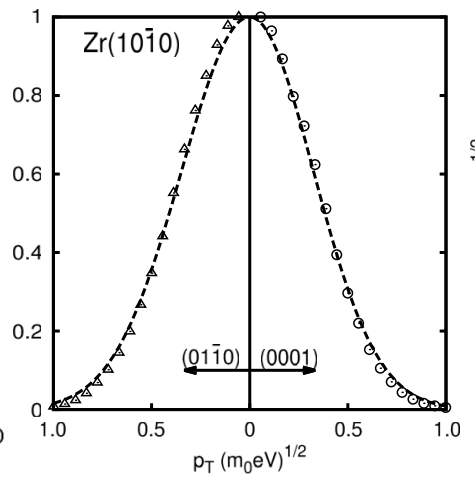

(b)

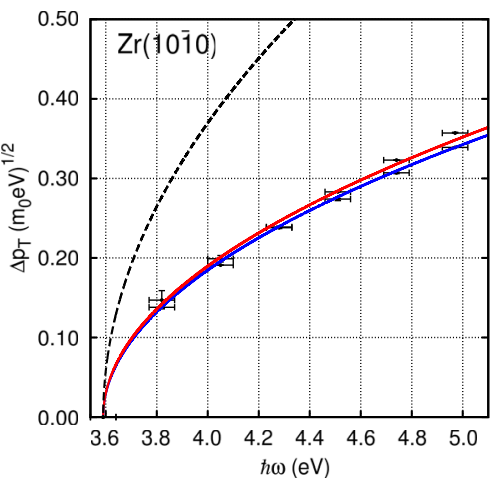

(c)

FIG. 2. Emission properties for the (1010) face of $\mathrm{Be}, \mathrm{Mg}, \mathrm{Y}$ and $\mathrm{Zr}$ metals. Column (a): Crystal momentum map of the electronic states (shaded regions) below the Fermi level (solid line) that may photoemit within $p_{T, \text { max }}=\sqrt{2 m_{0} \Delta E}$ (dashed lines) for the transverse (0001) and (0110) crystal directions. Column (b): Transverse momentum distributions of the photoemitted electrons in the (0001) and (0110) directions (Gaussian fits are guides to the eye). Column (c): Incident photon energy dependence of the rms transverse momentum $\Delta p_{T}$ for electron temperatures $T_{e} \rightarrow 0$ (data points with solid red line fit, for (011̄0) crystal direction and solid blue line fit for (0001) crystal direction), together with the expected form of $\Delta p_{T}(\hbar \omega)$ from Refs. [14] and [15] (dashed line). Black circles show the measured $\Delta p_{T}$ data from Refs. [34] and [36] for Be and $\mathrm{Mg}$, respectively. 
greater than that determined by our analysis. The DFTbased photoemission analysis is, however, consistent with the available experimental data for polycrystalline $\mathrm{Be}$ [34,35] and $\mathrm{Mg}$ [36] photocathodes, which are expected to preferentially expose (1010) microcrystalline faces [78]. For $\mathrm{Mg}$ with $\hbar \omega=4.66 \mathrm{eV}$, Wang et al. [36] report an upper limit on the normalized transverse emittance of $0.40( \pm 0.1) \mathrm{mm} \mathrm{mrad} / \mathrm{mm}$, which equates to $\Delta p_{T}=$ $0.290( \pm 0.07) \quad\left(m_{0} \mathrm{eV}\right)^{1 / 2}$-a value that is plotted in Fig. 2(c) for $\operatorname{Mg}(10 \overline{1} 0)$ emission with an estimated $\pm 0.1 \mathrm{eV}$ uncertainty in the effective work function due to both the uncertainty in the value of $\phi_{(10 \overline{1})}$ and the Schottky effect in the employed rf gun. Similarly, the span of the two directional transverse values of $\Delta p_{T}$ predicted for $\mathrm{Be}(10 \overline{10})$ emission is consistent with the value of $0.225( \pm 0.01)\left(m_{0} \mathrm{eV}\right)^{1 / 2}$ [also plotted in Fig. 2(c)] determined for a polycrystalline Be photocathode using the solenoid scan technique with a $20 \mathrm{kV}$ DC photo-gun and $4.75 \mathrm{eV}$ photons [34,35].

Table III summarizes the values of $A_{(0001)}$ and $A_{(01 \overline{1} 0)}$ (in terms of $\left.A_{(0001)}\right)$ extracted from the fits to the $\Delta p_{T}(\hbar \omega)$ DFT data in Fig. 2(c), together with results obtained for (1010)-face emission from hcp Hf, Sc, and Ti. The two Group IIa elements (Be and $\mathrm{Mg}$ ) are predicted to have the largest anisotropy in the rms transverse momentum of the emitted electrons, while emission from both the Group IIIb ( $\mathrm{Sc}$ and $\mathrm{Y}$ ) and Group IVb (Ti, Zr, and Hf) elements should be isotropic in $\Delta p_{T}$ to within $10 \%$ - a trend that is consistent with anisotropy associated with the photoemitting states [Fig. 2(a)]. The fact that all the values of $A_{(i j k l)}$ are around half of the $1 / \sqrt{3}$ factor derived in Refs. [14] and [15] suggests that these seven (1010)-face emitting hcp elemental metal photocathodes will have a brightness $\sim 4 \times$ larger than expected.

We also note that in accordance with the results for $\operatorname{Mg}(0001)$ emission (Sec. IV), the DFT-based photoemission analysis indicates that electron temperature $T_{e}$ has little effect on $\Delta p_{T}$ up to the melting points [77] of the four investigated hcp metal photocathodes. In particular, the values of $A$ in $\Delta p_{T}=A \sqrt{m_{0} \Delta E}$ do not change by more than about $1 \%$, which is well within the intrinsic uncertainty of the simulation technique. To a large extent, this

TABLE III. Photoemission properties of (1010)-face hcp metals.

\begin{tabular}{lccc}
\hline \hline & $\phi_{(10 \overline{1} 0)}( \pm 0.05 \mathrm{eV})$ & $A_{(0001)}$ & $A_{(01 \overline{1} 0)} / A_{(0001)}$ \\
\hline $\mathrm{Be}$ & 3.88 & 0.160 & 2.10 \\
$\mathrm{Mg}$ & 3.79 & 0.241 & 1.33 \\
$\mathrm{Sc}$ & 3.10 & 0.221 & 1.05 \\
$\mathrm{Y}$ & 3.08 & 0.222 & 1.08 \\
$\mathrm{Ti}$ & 3.63 & 0.295 & 1.04 \\
$\mathrm{Zr}$ & 3.59 & 0.287 & 1.03 \\
$\mathrm{Hf}$ & 3.63 & 0.281 & 1.08 \\
\hline \hline
\end{tabular}

temperature insensitivity is due to the preponderance of holelike states for (1010)-face emission, for which higher electron temperatures generate occupation in higher energy states with lower $p_{T}$ that therefore do not contribute to significant increase in $\Delta p_{T}$.

\section{VI. (0001)-FACE EMISSION}

Photoemission from the (0001) face of hcp metals generally produces a relatively isotropic rms transverse momentum distribution since the basal plane band dispersion is also quite isotropic, as shown for $\mathrm{Mg}$ (0001) in Fig. 1. There are, however, complications that are introduced by both the details of the electronic band structure, coupled with the required energy-momentum relationship [Eq. (1)], and the presence of two-dimensional surface states.

In contrast to $\mathrm{Mg}$, the Fermi level in Be and the Group IIIb metals ( $\mathrm{Sc}$ and $\mathrm{Y}$ ) does not cross the $\Gamma \rightarrow$ A direction of the Brillouin zone [79], meaning that there are no bulk electronic states that can photoemit electrons with zero transverse momentum $p_{T}$ for the (0001) emission direction. As a result, the values of $\phi_{(0001)}$ evaluated from the energetic difference between the vacuum and Fermi levels (Table II) will not reflect the actual bulk-state work function for the (0001) crystal face. The most extreme case is $\mathrm{Be}(0001)$ emission since, as shown in Fig. 3, the bottom of the occupied $\Gamma_{3}^{+}$band with an effective mass greater than the free electron mass $m_{0}$ [80] is $4.6 \mathrm{eV}$ below the Fermi level. As a result, the energy-momentum relationships governing the one-step photoemission process [Eq. (1)]

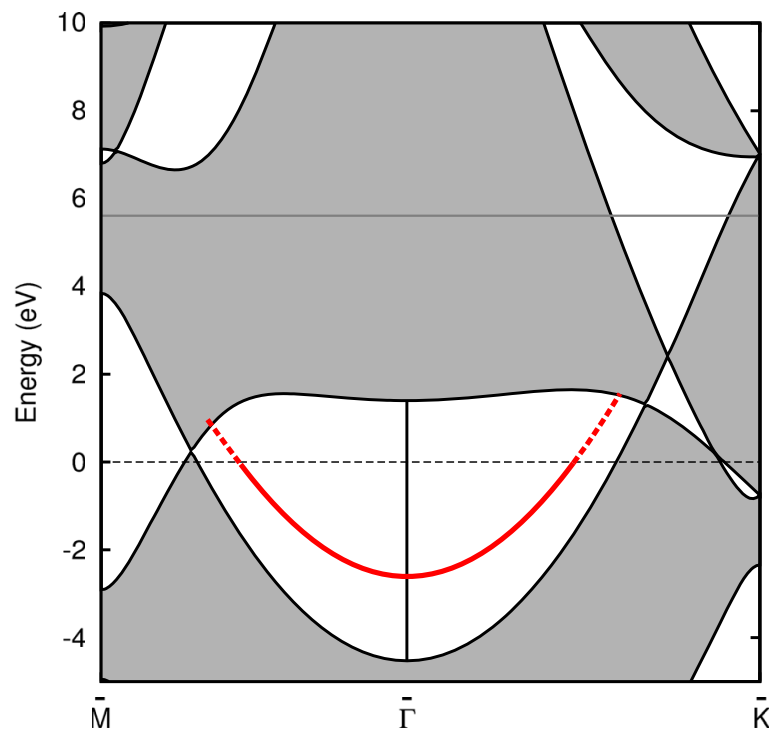

FIG. 3. Calculated Be(0001) bulk and surface electronic states in the surface Brillouin zone: Projected bulk states (grey shading) and the surface state below (solid red line) and above (dashed red line) the Fermi level (thin dashed line). The DFT evaluated vacuum level at $\phi_{(0001)}=5.61 \mathrm{eV}$ is indicated by a thin solid line. 
dictate that the first electrons to be emitted from the bulk states originate near the $\Gamma$ point and require a photon energy $\hbar \omega>\phi_{(0001)}+4.6 \mathrm{eV}$, giving an effective (0001)-face work function of about $10.2 \mathrm{eV}$ - an energy well beyond common UV laser sources. Similar arguments associated with the position of the occupied bands in Sc and $\mathrm{Y}$ indicate that their thin-slab calculated values of $\phi_{(0001)}$ are also increased to give effective work functions of around $4.55 \mathrm{eV}$ and $4.22 \mathrm{eV}$, respectively.

For sufficiently clean $\mathrm{Be}(0001)$ crystal faces, however, the effective work function will only be around $8.4 \mathrm{eV}$ due to the existence of a strong two-dimensional surface state in the $6 \mathrm{eV}$ energy gap between the lower occupied $\Gamma_{3}^{+}$ and upper unoccupied $\Gamma_{4}^{-}$bulk electronic bands [37-41]. This surface state, with its near-perfect parabolic dispersion about the $\bar{\Gamma}$ point of the surface Brillouin zone (SBZ), lies $1.8 \mathrm{eV}$ above the lower $\Gamma_{3}^{+}$bulk band at zone center and its $\bar{\Gamma}$ point is $2.8 \mathrm{eV}$ below the Fermi level [37-41]. Angle-resolved photoemission spectroscopy measurements [40] have indicated that the effective mass of the surface state is about $1.50 m_{0} ; m^{*}=1.53 m_{0}$ in the $\bar{\Gamma} \rightarrow \overline{\mathrm{M}}$ direction and $m^{*}=1.45 m_{0}$ in the $\bar{\Gamma} \rightarrow \overline{\mathrm{K}}$ direction. As for emission from the bulk states, this means that photoemission from the surface state of $\mathrm{Be}(0001)$ will first occur from states around the $\bar{\Gamma}$ point as this requires the least energy. Consequently, photoemission from the $\mathrm{Be}(0001)$ surface state is predicted to only occur when $\hbar \omega>\phi_{(0001)}+2.8 \mathrm{eV}$, where $\phi_{(0001)}=5.61 \mathrm{eV}$.

For the theoretical determination of the characteristics of the $\mathrm{Be}(0001)$ surface state within our DFT framework, thin-slab calculations employing the LDA pseudopotentials [43] in the Quantum-ESPRESSO code [42] are used. The surface is modeled in the two-dimensional irreducible SBZ with 13 atomic layers separated by $15 \AA$ of vacuum-the slab configuration used to obtain a reasonable value for the work function for the $\mathrm{Be}(0001)$ surface, which then sets the energy scale for the two-dimensional quantum-confined surface state. Figure 3 shows the results of the computed $\mathrm{Be}(0001)$ surface state electronic structure in the two transverse symmetry directions ( $\bar{\Gamma} \overline{\mathrm{M}}$ and $\bar{\Gamma} \overline{\mathrm{K}})$ of the SBZ along with the projected bulk states. The calculated minimum energy of the surface state (solid red line) at the $\bar{\Gamma}$ point is $2.83 \mathrm{eV}$ below the Fermi level (dashed line), which is in good agreement with the experimental value of $2.8( \pm 0.05) \mathrm{eV}$ [37-41]. Further, our evaluation of the $\mathrm{Be}(0001)$ surface state indicates that the dispersion of the confined state is quite isotropic with a transverse effective mass $m_{T}^{*} \approx 1.5 m_{0}$, in agreement with prior work [40]. At the $\bar{\Gamma}$ point, the surface state band is calculated to be $1.92 \mathrm{eV}$ above the minimum energy at the same crystal momentum of the lower bulk band $\Gamma_{3}^{+}$from which the electrons in the surface state originate [37-41]. This "zero point" energy $\Delta E_{s s}$ then allows evaluation of the longitudinal momentum of the electrons in the surface state; $p_{z}=\sqrt{2 m_{s s}^{*} \Delta E_{s s}} \approx 2.4\left(m_{0} \mathrm{eV}\right)^{1 / 2}$, if one assumes $m_{s s}^{*} \approx 1.5 m_{0}$ is also the effective electron mass of the surface state in the $z$ direction [i.e., the (0001) crystal direction].

With these assumed constant values of $p_{z}$ and $m_{z}$ for the emitting state, all required parameters in our DFT photoemission analysis are defined; in particular, those associated with the flux transmission probability over the work function barrier [Eq. (2)]. The predicted $p_{T}$ distribution of the emitted electrons for an excess energy $\Delta E=0.3 \mathrm{eV}$ [a photon energy $\hbar \omega=8.74 \mathrm{eV}(142 \mathrm{~nm})]$ is shown in Fig. 4. As $m_{T}^{*}>m_{0}$ and the surface state has a constant density of states $g(E, \vec{k})$, electrons are predominantly photoemitted from the $\bar{\Gamma}$ point and no electrons may be emitted beyond $p_{T, \text { max }}=\sqrt{2 m_{0} \Delta E} \approx 0.77\left(m_{0} \mathrm{eV}\right)^{1 / 2}$. In practice, $T\left(p_{z}, p_{z 0}\right)$ severely curtails emission beyond $p_{T}=0.5$ $\left(m_{0} \mathrm{eV}\right)^{1 / 2}$, resulting in a predicted value of $\Delta p_{T, \mathrm{Be}(0001)}=$ $0.141( \pm 0.05)\left(m_{0} \mathrm{eV}\right)^{1 / 2}$. As expected from the isotropic dispersion of the two-dimensional surface state, the $p_{T}$ distribution is also symmetric with respect to $p_{T}=0$.

Surface states also exist on the (0001) face of other hcp metals [81,82], but are less robust than that of $\mathrm{Be}(0001)$. In the case of $\operatorname{Mg}(0001)$ [83,84], the Fermi level cuts the upper $\Gamma_{4}^{-}$band ensuring that there are filled bulk states around the $\bar{\Gamma}$ point of the SBZ which contribute to and dominate the photoemission as shown in Fig. 1. For Sc and Y, our DFT-based analysis indicates that clean (0001) crystal surfaces of these elemental metals should also have a surface state. To our knowledge, however, there have been no experimental studies on either $\mathrm{Sc}(0001)$ or $\mathrm{Y}(0001)$ to compare with and verify our calculations.

The simulation of bulk state photoemission from the (0001) faces of the Group IVb metals ( $\mathrm{Ti}, \mathrm{Zr}$, and $\mathrm{Hf}$ ) is complicated, much more so than for their (1010) faces, by

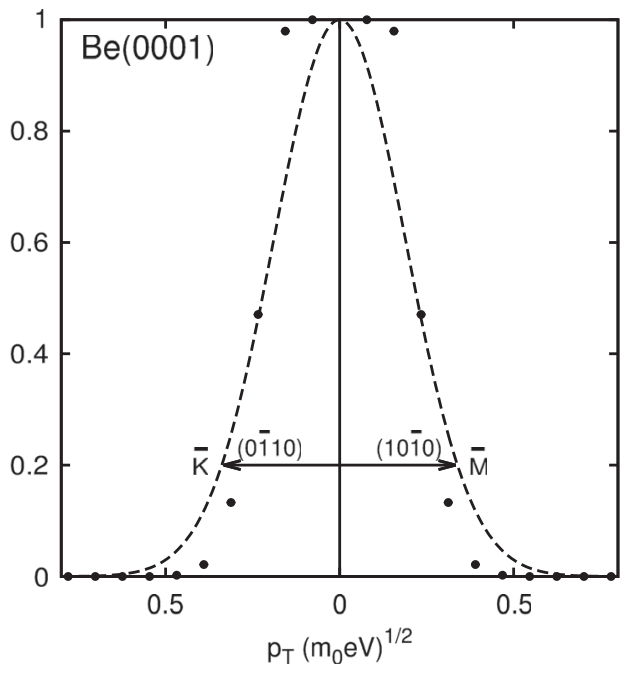

FIG. 4. Evaluated transverse electron momentum distributions (solid circles) in the $\overline{\mathrm{M}}$ and $\overline{\mathrm{K}}$ directions of the surface Brillouin

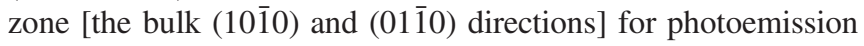
from the $\mathrm{Be}(0001)$ surface state with $\hbar \omega=8.74 \mathrm{eV}$. [Gaussian fit is guide to the eye (dashed line).] 
the positioning of the Fermi level in the DFT evaluated band structure. The reason for this is the close proximity of the $\Gamma_{3}^{+}$band to the Fermi level in the $\Gamma \rightarrow$ A direction; that is, whether or not the $\Gamma_{3}^{+}$band cuts the Fermi level in this direction. Our DFT calculations suggest that it does not, which results in there being no emitting bulk states with $p_{T}=0$. On the other hand, the work of D. A. Papaconstantopoulos [79] indicates that it does, while the Fermi surface database from the University of Florida [85] shows a Fermi surface along the $\Gamma \rightarrow \mathrm{A}$ direction for $\mathrm{Ti}$ and $\mathrm{Hf}$, but not for $\mathrm{Zr}$. As the one-step photoemission process is sensitive to the details of the electronic band structure near the Fermi level, either further more accurate theoretical calculations or, ideally, detailed ARPES (or $\Delta p_{T}$ ) measurements are needed to determine the photoemission properties of the (0001) faces of the Group IIIb metals.

\section{SUMMARY}

The presented DFT-based analysis of the properties of photocathodes clearly indicates that the rms transverse momentum $\Delta p_{T}$ of electrons photoemitted in a one-step quantum mechanical process [86,87] is fundamentally dependent upon the dispersive nature of the electronic bands from which they originate. This is shown to be particularly true of the hop metals - the most common crystal structure amongst the elemental metals-since the crystal symmetry, and hence electronic band structure, is intrinsically anisotropic. Whereas emission from the basal plane, the (0001)-face, usually generates an isotropic transverse momentum distribution (Fig. 1) due to hexagonal face symmetry, emission from the generally most prevalent (1010)-face in polycrystalline hcp metals can be quite anisotropic (Fig. 2). As the rms transverse momentum $\Delta p_{T}$ is also dependent on the excess photoemission energy, knowledge of the work function for each crystal emission face, $\phi_{(i j k l)}$, is also critical to the DFTbased photoemission simulation. A standard thin-slab evaluation method for $\phi$ [33], which employs the atomic crystal potential from the band structure calculations, is shown to provide values in good agreement with literature values. The average of the resulting anisotropic values of $\Delta p_{T}$ obtained from the theoretical simulation for the most prevalent (1010)-face of $\mathrm{Be}$ and $\mathrm{Mg}$ are consistent with experimental measurements [34-36].

Emission from the strong surface state of the $\mathrm{Be}(0001)$ face [37-41] is also analyzed. As the effective mass $m^{*}$ associated with the dispersion of this two-dimensional state is greater than $m_{0}$ and fairly symmetric, the transverse momentum distribution of the photoemitted electrons is maximized at and quite symmetric about $p_{T}=0$ (the $\bar{\Gamma}$ point of the surface Brillouin zone). If its effective mass were less than $m_{0}$, the one-step analysis would predict emission peaked at $p_{T}=\sqrt{2 m^{*} E_{F}}$, which may then directly produce an electron beam suitable for hollow cone illumination
[88-90]. The use of such a state can be dependent upon the cleanliness of the laser-driven electron gun since chemical contamination of the photocathode surface may destroy environmentally sensitive surface states. Similar considerations also affect the use of bulk elemental hcp metal photocathodes and together with impractical high work functions, low melting points, and magnetic effects effectively restrict the choice to only a handful of elemental hcp metals: Be, Mg, Sc, Ti, Y and Zr. Bi-metal hep compounds may well overcome many such issues.

\section{ACKNOWLEDGMENTS}

The authors are indebted to Juan Carlos Campuzano, Christopher Grein, Randall Meyer, and Serdar Öğüt for their valuable discussions. This work was partially supported by the Department of Energy (Contract No. DEFG52-09NA29451).

[1] Z. Huang and K.-J. Kim, Phys. Rev. ST Accel. Beams 10, 034801 (2007).

[2] K. Németh, K. C. Harkay, M. van Veenendaal, L. Spentzouris, M. White, K. Attenkofer, and G. Srajer, Phys. Rev. Lett. 104, 046801 (2010).

[3] V. A. Lobastov, R. Srinivasan, and A. H. Zewail, Proc. Natl. Acad. Sci. U.S.A. 102, 7069 (2005).

[4] M. R. Armstrong, B. W. Reed, B. R. Torralva, and N. D. Browning, Appl. Phys. Lett. 90, 114101 (2007).

[5] O. Bostanjoglo and R. Liedtke, Phys. Status Solidi A 60, 451 (1980).

[6] H. Dömer and O. Bostanjoglo, Rev. Sci. Instrum. 74, 4369 (2003).

[7] M. Reiser, Theory and Design of Charged Particle Beams (John Wiley \& Sons, New York, 2008).

[8] C. D. Child, Phys. Rev. (Series I) 32, 492 (1911).

[9] I. Langmuir, Phys. Rev. 21, 419 (1923).

[10] T. Srinivasan-Rao, J. Fischer, and T. Tsang, J. Appl. Phys. 69, 3291 (1991).

[11] T. Anderson, I. V. Tomov, and P. Rentzepis, J. Appl. Phys. 71, 5161 (1992).

[12] A. Valfells, D. W. Feldman, M. Virgo, P. G. O'Shea, and Y. Y. Lau, Phys. Plasmas 9, 2377 (2002).

[13] I. V. Bazarov, B. M. Dunham, and C. K. Sinclair, Phys. Rev. Lett. 102, 104801 (2009).

[14] D. H. Dowell and J. F. Schmerge, Phys. Rev. ST Accel. Beams 12, 074201 (2009).

[15] K. L. Jensen, P. G. O'Shea, D. W. Feldman, and J. L. Shaw, J. Appl. Phys. 107, 014903 (2010).

[16] J. Simmons, Phys. Rev. Lett. 15, 967 (1965).

[17] R. W. Stark and L. M. Falicov, Phys. Rev. Lett. 19, 795 (1967).

[18] V. Fomenko, Powder Metallurgy and Metal Ceramics 33, 85 (1995).

[19] M. Methfessel, D. Hennig, and M. Scheffler, Phys. Rev. B 46, 4816 (1992).

[20] Y. Morikawa, H. Ishii, and K. Seki, Phys. Rev. B 69, 041403 (2004).

[21] N. D. Lang and W. Kohn, Phys. Rev. B 3, 1215 (1971). 
[22] R. Klein, Surf. Sci. 20, 1 (1970).

[23] V. Sahni, J. P. Perdew, and J. Gruenebaum, Phys. Rev. B 23, 6512 (1981).

[24] H. L. Skriver and N. M. Rosengaard, Phys. Rev. B 46, 7157 (1992).

[25] R. C. Jerner and C. B. Magee, Oxid. Met. 2, 1 (1970).

[26] R. G. Wilson, J. Appl. Phys. 37, 2261 (1966).

[27] A. K. Green and E. Bauer, Surf. Sci. 74, 676 (1978).

[28] F. Batallan, I. Rosenman, and C. B. Sommers, Phys. Rev. B 11, 545 (1975).

[29] Y. Ishizawa and W. R. Datars, Phys. Rev. B 2, 3875 (1970).

[30] P. Chatterjee, Phys. Rev. B 27, 4722 (1983).

[31] N. Lang and W. Kohn, Phys. Rev. B 3, 1215 (1971).

[32] H. L. Skriver and N. M. Rosengaard, Phys. Rev. B 46, 7157 (1992).

[33] C. J. Fall, N. Binggeli, and A. Baldereschi, J. Phys. Condens. Matter 11, 2689 (1999).

[34] B. L. Rickman, J. A. Berger, A. W. Nicholls, and W. A. Schroeder, Phys. Rev. Lett. 111, 237401 (2013).

[35] B. L. Rickman, J. A. Berger, A. W. Nicholls, and W. A. Schroeder, Phys. Rev. Lett. 113, 239904(E) (2014).

[36] X. J. Wang, M. Babzien, R. Malone, and Z. Wu, in Proceedings of the European Particle Accelerator Conference, Vienna, 2000 (EPS, Geneva, 2000).

[37] P. J. Feibelman, Phys. Rev. B 46, 2532 (1992).

[38] R. A. Bartynski, E. Jensen, T. Gustafsson, and E.W. Plummer, Phys. Rev. B 32, 1921 (1985).

[39] K. Pohl, J.-H. Cho, K. Terakura, M. Scheffler, and E. W. Plummer, Phys. Rev. Lett. 80, 2853 (1998).

[40] J. C. Boettger and S. B. Trickey, Phys. Rev. B 34, 3604 (1986).

[41] P. T. Sprunger, L. Petersen, E. W. Plummer, E. Lægsgaardgsgaard, and F. Besenbacher, Science 275, 1764 (1997).

[42] P. Giannozzi et al., J. Phys. Condens. Matter 21, 395502 (2009).

[43] K. F. Garrity, J. W. Bennett, K. M. Rabe, and D. Vanderbilt, Comput. Mater. Sci. 81, 446 (2014).

[44] J. P. Perdew, K. Burke, and M. Ernzerhof, Phys. Rev. Lett. 77, 3865 (1996).

[45] H. J. Monkhorst and J. D. Pack, Phys. Rev. B 13, 5188 (1976).

[46] N. Marzari, D. Vanderbilt, A. De Vita, and M. C. Payne, Phys. Rev. Lett. 82, 3296 (1999).

[47] O. Jepsen, O. K. Andersen, and A. R. Mackintosh, Phys. Rev. B 12, 3084 (1975).

[48] P. Soven, Phys. Rev. 137, A1706 (1965).

[49] C. M. Singal and T. P. Das, Phys. Rev. B 16, 5068 (1977).

[50] T. L. Loucks and P. H. Cutler, Phys. Rev. 133, A819 (1964).

[51] H. J. Gotsis, D. A. Papaconstantopoulos, and M. J. Mehl, Phys. Rev. B 65, 134101 (2002).

[52] L. F. Mattheiss, Phys. Rev. 151, 450 (1966).

[53] S. L. Altmann and C. J. Bradley, Proc. Phys. Soc. London 92, 764 (1967).

[54] C. Kittel and P. McEuen, Introduction to Solid State Physics, Vol. 8 (Wiley, New York, 1976).

[55] P. M. Holtham, J.-P. Jan, and H. L. Skriver, J. Phys. F 7, 635 (1977).

[56] R. A. Deegan, Phys. Rev. 171, 659 (1968).

[57] D. G. Pettifor, J. Phys. F 7, 613 (1977).
[58] C. J. Fall, N. Binggeli, and A. Baldereschi, J. Phys. Condens. Matter 11, 2689 (1999).

[59] N.E. Singh-Miller and N. Marzari, Phys. Rev. B 80, 235407 (2009).

[60] S. Chen, Surf. Sci. 264, L162 (1992).

[61] M. Green, Solid State Surface Science (M. Dekker, New York, 1969), Vol. 1.

[62] H. B. Michaelson, J. Appl. Phys. 48, 4729 (1977).

[63] E. V. Chulkov, V. M. Silkin, and E. N. Shirykalov, Surf. Sci. 188, 287 (1987).

[64] E. Sobczak, P. O. Nilsson, and K. Karlsson, Acta Phys. Pol. Ser. A 82, 337 (1992).

[65] J. Lahtinen, J. Vaari, and K. Kauraala, Surf. Sci. 418, 502 (1998).

[66] H. Kawano, Prog. Surf. Sci. 83, 1 (2008).

[67] F. J. Himpsel, K. Christmann, P. Heimann, D. E. Eastman, and P. J. Feibelman, Surf. Sci. 115, L159 (1982).

[68] D. M. Hanson, R. Stockbauer, and T. E. Madey, Phys. Rev. B 24, 5513 (1981).

[69] M. Yamamoto, C. T. Chan, and K. M. Ho, Phys. Rev. B 50, 7932 (1994).

[70] S. Karkare and I. Bazarov, Appl. Phys. Lett. 98, 094104 (2011).

[71] G. Lee, Fermilab Report No. TM-1615, 1980.

[72] G. W. Mahlman, J. Appl. Phys. 20, 197 (1949).

[73] J. A. Berger, B. L. Rickman, T. Li, A. W. Nicholls, and W. A. Schroeder, Appl. Phys. Lett. 101, 194103 (2012).

[74] J. Chen, D. Tzou, and J. Beraun, Int. J. Heat Mass Transfer 49, 307 (2006).

[75] E. Jeon, J. Kim, Y. Kim, K. Ma, and J. Nam, Nucl. Instrum. Methods Phys. Res., Sect. A 697, 46 (2013).

[76] J. C. Kimball, R. W. Stark, and F. M. Mueller, Phys. Rev. 162, 600 (1967).

[77] W. M. Haynes, CRC Handbook of Chemistry and Physics (CRC press, Boca Raton, FL, 2013).

[78] U. F. Kocks, C. N. Tomé, and H.-R. Wenk, Texture and Anisotropy: Preferred Orientations in Polycrystals and Their Effect on Materials Properties (Cambridge University Press, Cambridge, England, 2000).

[79] D. A. Papaconstantopoulos, Handbook of the Band Structure of Elemental Solids (Springer, New York, 1986).

[80] J. H. Tripp, P. M. Everett, W. L. Gordon, and R. W. Stark, Phys. Rev. 180, 669 (1969).

[81] P. J. Feibelman and D. Hamann, Solid State Commun. 31, 413 (1979).

[82] R. I. R. Blyth, P. T. Andrews, and S. D. Barrett, J. Phys. Condens. Matter 3, 2827 (1991).

[83] E. Chulkov, V. Silkin, and E. Shirykalov, Surf. Sci. 188, 287 (1987).

[84] U. O. Karlsson, G. V. Hansson, P. E. S. Persson, and S. A. Flodström, Phys. Rev. B 26, 1852 (1982).

[85] T. Choy, J. Naset, J. Chen, S. Hershfield, and C. Stanton, Bull. Am. Phys. Soc. 45, L36 (2000).

[86] G. Mahan, Phys. Rev. B 2, 4334 (1970).

[87] R. Courths and S. Hüfner, Phys. Rep. 112, 53 (1984).

[88] W. Krakow and L. A. Howland, Ultramicroscopy 2, 53 (1976).

[89] C. Dinges, H. Kohl, and H. Rose, Ultramicroscopy 55, 91 (1994).

[90] Y. Kondo, T. Ito, and Y. Harada, Jpn. J. Appl. Phys. 23, L178 (1984). 